\title{
Determination and Comparative Study of Sugars and Synthetic Colorants in Commercial Branded Fruit Juice Products
}

\author{
S. D. C. Sewwandi, ${ }^{1}$ P. C. Arampath, ${ }^{1}$ A. B. G. Silva $\mathbb{D},{ }^{2}$ and R. Jayatissa ${ }^{2}$ \\ ${ }^{1}$ Department of Food Science and Technology, Faculty of Agriculture, University of Peradeniya, Peradeniya, Sri Lanka \\ ${ }^{2}$ Department of Nutrition, Medical Research Institute, Colombo 08, Sri Lanka \\ Correspondence should be addressed to A. B. G. Silva; buddhika-gayani@mri.gov.lk
}

Received 6 June 2019; Revised 4 December 2019; Accepted 16 January 2020; Published 7 February 2020

Academic Editor: Antoni Szumny

Copyright (c) 2020 S. D. C. Sewwandi et al. This is an open access article distributed under the Creative Commons Attribution License, which permits unrestricted use, distribution, and reproduction in any medium, provided the original work is properly cited.

\begin{abstract}
Fruit juice-based products are potentially high demanded products in Sri Lanka. The research was conducted to estimate the total sugar content in commercially available fruit juice products and to compare the suitability and efficiency of analytical methods: Lane and Eynon titration method and UV-Visible spectrophotometric methods. Further synthetic colorants in the products were identified and compared with the label information. A consumer survey was conducted to elicit information on consumer preference and knowledge on sugar content of the products. Based on the survey, consumers were more concerned about taste (37\%), brand name (28\%), price (28\%), and nutrition value (7\%), respectively. Awareness about the Recommended Dietary Allowance (RDA) of sugar, added sugar consumption, and risk of chronic diseases was $49 \%$. Awareness on the color coding system of the products was $68 \%$. The total sugar content of the fruit nectars was determined using both methods. The maximum total sugar level $(18.38 \mathrm{~g} / 100 \mathrm{~mL})$ was observed by the titration method, while the maximum total sugar level $(18.31 \mathrm{~g} / 100 \mathrm{~mL})$ was measured by the spectrophotometric method in wood apple (Limonia acidissima) nectar in brand No. "1." The maximum sucrose content $(10.57 \mathrm{~g} / 100 \mathrm{~mL})$ was observed in mango (Mangifera indica L.) nectar in the brand No. "1." Total sugar contents of both tested methods were compared using SAS 9.0 (Randomized Complete Block Design, RCBD). There is no significant difference $(P>0.05)$ between the two testing methods. In conclusion, the Lane and Eynon titration method was identified as the most effective method for analysis of the total sugar in fruit juice-based products. Based on the analysis of thin-layer chromatography (TLC), a majority of fruit nectar samples (57.14\%) contained natural colorants, while $42.86 \%$ of total samples contained synthetic colorants. Labelling violation was done by the manufacturers, and the consumer awareness was high with the color-coding system. Continuous surveillance is recommended to minimize health risk related to high sugar consumption of the population.
\end{abstract}

\section{Introduction}

The annual growth rate of fruit-based beverage products (FBBP) in Sri Lanka is $12 \%$ and worth of 12 million US\$. The consumer demands for fruit juice beverage products have been increased due to awareness among the public on health promoting compounds, health benefits, and balanced diet [1]. Fruit juices contain substantial amount of vitamins, minerals, antioxidants, and phytochemicals. Thus, they fulfill the daily requirement of nutrients. They transform human health with a wellness technique that improves their immune system and cleanses human body of harmful toxins [2]. All monosaccharides and disaccharides in food are defined as "Sugar" [3]. Recommended Dietary Allowance (RDA) of sugar is $25 \mathrm{~g}$, based on the World Health Organization (WHO) [4].

The price of the fruit juices (100\% pure) is higher than the sugar added beverages, namely, fruit nectars and undiluted/diluted forms of squashes. A single serving of these types of fruit juice products possesses the amount of sugars exceeding the RDA value of sugars for an adult [2]. Therefore, the health benefits of these sweetened fruit juice beverages are lower than the pure fruit juices. The comparative price of these sweetened fruit juice beverages is lower, and the demand and quality are highly varied. 
In Sri Lanka, over $40 \%$ of population are prediabetic or diabetic [5]. Prediabetic patients are diagnosed and directed to the clinics for advice and treatments. A diet with high sugar and carbohydrates promotes diabetes condition [5]. As the first step to combat diabetic epidemic, the Ministry of Health, Sri Lanka, introduced "the Traffic Lights Color Coding system (August 2016), under Section 32 of the Food Act No. 26 of 1980. Based on this regulation, color codes with sugar percentage should be included in the label by the manufacturers. Color code of the label is red for the sugar content over $11 \mathrm{~g}$ per $100 \mathrm{~mL}$. The color code of the label is amber or yellow/amber for the sugar content $2 \mathrm{~g}-11 \mathrm{~g}$ per $100 \mathrm{~mL}$, and the color code green is for the sugar content below $2 \mathrm{~g}$ per $100 \mathrm{~mL}$ [3].

Food colorant can be defined as a dye, pigment, or any other substance that provides color when it is added to a food or beverage. It is a food additive present in different forms such as liquid, gel, paste, and powder. It can be divided into 4 major categories as natural, nature identical, synthetic, and inorganic [6]. Synthetic food colorants are substances of chemicals which do not occur in nature and have been made in factory. These colorants are usually water-soluble and can be used in foods without any further processing [7].

Food colorants are the major source of food intoxication and surveys have been conducted to determine the presence of nonpermitted food colors in different food products [7]. The elimination of most food dyes is just due to their toxicological, mutagenic, and carcinogenic properties. One example for the harmful dye is Sudan: causing mutagenic changes and damaging stomach [8]. Furthermore, colorants may be associated with various complications such as asthma, urticaria, abortion, hyperactivity of children, carcinogenicity, decreased intelligence quotient (IQ) of children, anaphylactic reactions, idiosyncrasy, weakening of the immune system, decreased WBC and lymphocyte count, and vitamin B6 deficiency [9, 10].

The maximum limit of permissible colorants to be added in any food shall be $0.1 \mathrm{~g} / \mathrm{kg}$ of food as consumed. The Acceptable Daily Intake (ADI) has been defined as the amount of a substance that can be consumed everyday throughout the lifetime of an individual without any appreciable health effects. Even permissible artificial colorants, if consumed indiscriminately, are not safe. The ADI of erythrosine was reduced from 2.5 to $0.1 \mathrm{mg} \cdot \mathrm{kg}^{-1}$ body weight, as it produced effects on thyroid function in shortterm studies in rats [11].

According to the Food Act No. 26 of 1980 in Sri Lanka, Extra ordinary gazette No. 1688/28, 2011, only nine synthetic colorants are permitted and the maximum limit is given as $100 \mathrm{mg}$ per $1 \mathrm{~kg}$ as a single component or combination. Permitted colors are Carmoisine/Azorubine (E122), Ponceau 4R (E124), Erythrosine (E127), Allura red (E129), Tartrazine (E102), Sunset yellow FCF (E110), Indigotine/ Indigo Carmine (E132), Brilliant Blue FCF (E133), and Fast Green FCF (E143) [12].

The objective of the research was to investigate the consumer preference, content of sugars, and synthetic colorants used in the commercial branded fruit juice products in Sri Lanka and the label violations according to the Food Act No. 26 of 1980 in Sri Lanka.

\section{Materials and Methods}

Fruit nectars and fruit cordials are two categories of FBBP available in the market. This study consists of three components: a consumer survey to assess the knowledge and preferences of FBBP; analyze sugar content and presence of artificial colorants in FBBP; and assess the awareness of color-coding labelling information on sugar.

2.1. Consumer Survey. This research includes analyzing cross-sectional data gathered through a consumer survey. A self-administered questionnaire with closed ended questions was used to gather information on frequency of consumption of FBBP, consumer preference of the fruit juice products, amount usually consumed at one time, level of awareness, and opinion about the color coding system for sugar introduced by the food regulatory authority in Sri Lanka.

2.1.1. Development and Validation of the Questionnaire. The development and validation of the questionnaire was done using the exploratory survey. An exploratory survey was conducted among school children, undergraduates, and adults (employees and nonemployees) from Colombo district area. Based on the results of the explorative survey, two forms of questionnaires were developed. One was a Google form [13] to be posted in the social media platform for school children and undergraduate students. Second was a paper-based questionnaire to be administered among adults. Google questionnaire was posted in the social media for 3 months and paper-based questionnaire was distributed among convenient sample of adults all over the country.

2.1.2. Study Area and Population. The survey was conducted within the country, Sri Lanka, as the survey was targeted on FBBP consumption in the whole country. The target population of the research was identified using an exploratory survey according to the objectives of the research. So, school children, university students, and adults (employees and nonemployees) who are included in the age range from 15 to 50 years or above were used. A total sample of 220 people was selected from island wide and random sampling process was applied.

2.1.3. Data Collection and Analysis of Questionnaires. Data were collected by using both online-based [13] and paper-based questionnaire. Prior to distributing the questionnaire, a description of the survey and its importance were provided and the respondents were assured of the confidentiality of the data gathered. By using questionnaires, data about consumers' most preferable FBBP brand, product type, frequency of consumption, amount consumed at once, awareness, and knowledge were collected. Furthermore, opinions of the consumers about color coding system for 
sugar level were gathered. A descriptive analysis was carried out to define and explain the results. The results of the survey were summarized to identify the above beverages consumption patterns of the study population. The most frequently consuming brands and product types were identified using the results of the survey.

2.2. Selection of Branded Fruit Juice Products. The research was targeted on beverage products that are currently advertising the color coding system (traffic light system) for sugar level in the label. Based on the survey, fruit nectar was reported the highest interested category of juice products. Therefore, fruit juice nectar products were used for the investigation. Currently, the color coding system for sugar level has not been implemented for cordial products. Cordial products were selected for the survey because the information provided by the survey would be useful for future implementation of color-coding system for cordial products in Sri Lanka.

2.2.1. Sampling Procedure of Fruit Nectar. A total of 4 brands of fruit nectar samples were purchased based on the higher consumer preference. Among fruit nectar types, mixed fruit, mango, orange, and wood apple were the most preferred types. From brand 1 and brand 3, all four types were available. In brand 2 , orange type and in brand 4 , wood apple type was not available in the market. Therefore, a total of 14 products were purchased from 4 brands for the laboratory analysis. In each type, 4 samples from different batches were taken for the analysis. Therefore, a total of 56 samples in triplicate were analyzed in the laboratory.

2.3. Chemicals and Instruments Used for the Research. Fehling A solution, Fehling B solution, methylene blue aqueous solution, glucose (AR grade), sucrose (AR grade), $\mathrm{NaOH}$ (VWR Prolabo Chemicals, Sussex, England), phenolphthalein indicator, $\mathrm{HCl}$ (Pharmacos, Sussex England), $n$-butanol (Pharmacos, Sussex England, Batch No. 7544), $2 \mathrm{M}$ ammonia solution (VWR Prolabo Chemicals, Sussex, England), $2 \mathrm{M}$ acetic acid solution (VWR Prolabo Chemicals, Sussex, England), standard color sample (Carmosine, Tartrazine, Allula red, Sunset yellow, Ponceau 4R, Metanil yellow, Sudan 1, Sudan 2, Sudan 3, and Sudan 4), glacial acetic acid (VWR Prolabo Chemicals, Sussex, England), distilled water, conc. sulfuric acid, and 5\% phenol solution were used for sugar and colorant analysis.

UV-visible spectrophotometer (Genesys 10S UV-visible spectrophotometer), silica-coated aluminium TLC plates (Sigma-Aldrich), and Brix meter (ATAGO N-1E, Brix 0 32\%) were used.

2.4. Analysis for Sugar Content. Sugar content was quantified using both the Lane and Eynon method and spectrophotometric method (colorimetric method) for all the samples.

2.4.1. Lane and Eynon Titration Method. This method is one of the titration methods for determining the concentration of reducing sugars in a sample based on their reducing action towards certain metallic salts. Since sucrose is a nonreducing sugar, sucrose was converted into monosaccharides to get the reducing activity. In this process, the sample reduces copper sulfate in an alkaline tartrate system (Fehling's solution). AOAC Official Method 923.09, 2005, followed [14].

(1) Materials and Reagents.

Fehling's "A" solution: dissolved $34.64 \mathrm{~g} \mathrm{CuSO}_{4} \cdot 5 \mathrm{H}_{2} \mathrm{O}$ in Milli-Q water, diluted to $500 \mathrm{~mL}$, and filtered through glass wool.

Fehling's "B" solution: dissolved $173 \mathrm{~g}$ potassium sodium tartrate $4 \mathrm{H}_{2} \mathrm{O}$ and $50 \mathrm{~g} \mathrm{NaOH}$ in Milli-Q water, diluted to $500 \mathrm{~mL}$, kept for 2 days, and filtered through a $0.45 \mu \mathrm{m}$ filter paper.

Methylene blue indicator solution: $0.1 \mathrm{~g}$ of methylene blue $\left(\mathrm{C}_{16} \mathrm{H}_{18} \mathrm{ClN}_{3} \mathrm{~S} \cdot 2 \mathrm{H}_{2} \mathrm{O}\right)$ dissolved in $100.00 \mathrm{~mL}$ Milli-Q water.

Invert sugar standard solution: $9.50 \mathrm{~g}$ pure sucrose, $100 \mathrm{~mL}$ Milli-Q water, and $5 \mathrm{ml}$ of conc. HCl. Stored for 3 days at $20-25^{\circ} \mathrm{C}$ and then diluted to $1 \mathrm{~L}$. Neutralized with $1 \mathrm{M} \mathrm{NaOH}$ immediately before use.

(2) Standardization of Sucrose Solution.10\% dilute invert sugar standard solution was prepared and the $\mathrm{pH}$ was adjusted to 8.0. It was titrated with $10.0 \mathrm{~mL}$ of boiling Fehling's A and Fehling's B solution. The end point was determined by mixing $1 \%$ methylene blue until the blue color of the indicator disappeared to a brick red end point. The titration was completed within 3 minutes and blank titration was performed without hydrolyzing sucrose sample. The titration was performed in triplicates and the average value was taken.

(3) Determination of Initial Reducing Sugar Content. Accurately $5 \mathrm{~mL}$ of fruit juice sample was measured and 50 times diluted solution was prepared and the $\mathrm{pH}$ was adjusted to 8.0. This solution was transferred into a burette. Titration was performed as the above procedure.

(4) Hydrolysis of the Fruit Juice Sample and Analysis of Total Sugar Content. About $5 \mathrm{~mL}$ of fruit juice sample was mixed with $3 \mathrm{~mL}$ of conc. $\mathrm{HCl}$ and it was kept at $68^{\circ} \mathrm{C}$ for 30 minutes. The $\mathrm{pH}$ of the mixture was adjusted to 8.0 , and 50 times diluted solution was prepared using this hydrolyzed sample. This solution was transferred into a burette. Titration was performed as the above procedure.

(5) Method Validation and Quality Control. Three tested samples were spiked with the known volume of invert sugar standard solution and the titration was carried out as above and the final results were taken. Furthermore, these three samples were spiked with the known amount of sucrose and the titration was carried out and results were taken.

(6) Calculation.

$$
\begin{aligned}
\text { Sugar content }\left(\mathrm{g}_{\left.100 \mathrm{~mL}^{-1}\right)=}\right. & {[E+(A B-C)] * \frac{1}{D} } \\
& * \text { dilution factor } * \frac{100}{1000},
\end{aligned}
$$


where weight of the standard sucrose $=A \mathrm{mg} / \mathrm{mL}$. Required volume of hydrolyzed standard sucrose solution to titrate with Fehling $A$ and $B=B \mathrm{~mL}$. Calculated dextrose factor $=(A * B) \mathrm{mg}$. Dextrose factor from table $=C \mathrm{mg}$. Correction factor $=(A * B)-C \mathrm{mg}$. Required volume of sample to titrate with Fehling $A$ and $B=D \mathrm{~mL}$. Dextrose factor of sample from table $=E \mathrm{mg}$.

(7) Determination of Sucrose Content in Fruit Juice Samples. Total reducing sugar content of initial fruit juice sample was quantified by titration. Then total reducing sugar content of the hydrolyzed fruit juice sample was quantified in the same manner. Then, the total reducing sugar content of the hydrolyzed fruit juice sample and reducing sugar content of original fruit juice sample were subtracted and the value was taken as $\% \mathrm{w} / \mathrm{w}$. Then this value was multiplied by 0.95. Sucrose content of the sample was obtained through this calculation as $\% \mathrm{w} / \mathrm{w}$. These nonreducing sugars are usually expressed in terms of sucrose and $0.95 \mathrm{~g}$ sucrose on hydrolysis yields $1 \mathrm{~g}$ invert sugar (glucose + fructose). Factor 0.95 was used for the calculation. Then sucrose percentage was multiplied by weight $(\mathrm{g})$ of the $100 \mathrm{~mL}$ fruit juice sample. Sucrose content can be observed as g/100 mL:

sucrose in the sample $(\% \mathrm{w} / \mathrm{w})=[$ total reducing sugars $(\% \mathrm{w} / \mathrm{w})-$ initial reducing sugars $(\% \mathrm{w} / \mathrm{w})] * 0.95$.

In this method, reducing sugar solution was used to titrate a fixed volume of standard copper sulfate in alkaline tartrate (Fehling) solution to methylene blue end point. All the calculations were done according to the reference table (invert sugar table for $10 \mathrm{~mL}$ of Fehling's solution). To avoid the effect of errors happening during the whole procedure, standard sucrose solution $(1.9 \mathrm{mg} / \mathrm{mL})$ was used and a correction factor was developed with comparing both calculated value and the table value of standard sugar content. This correction factor was used for the further calculations of fruit nectars samples. The whole titration procedure was done under the boiling condition and titration should be completed within 3 minutes because it takes more than 3 minutes for the back reaction to take place [14]. End point of the titration should be determined very carefully. For high precision, titration was done in triplicate.

\subsubsection{Determination of Total Sugar Level in Fruit Nectars Using UV-Visible Spectrophotometric Method.}

(1) Sample Preparation and Analysis of Sugar Content in Standard Solution. About $1 \mathrm{~mL}$ of each standard was mixed with $1 \mathrm{~mL}$ of $5 \%$ aqueous solution of phenol and $5 \mathrm{~mL}$ of concentrated sulfuric acid. It was placed in $100^{\circ} \mathrm{C}$ water bath for 5 minutes and vortexed for 30 seconds for color development. Reference solutions were prepared in identical manner as above, except that $1 \mathrm{~mL}$ of sample was replaced by distilled water. Then the absorbance of the standard was measured by UV-visible spectrophotometer (Genesys10S UV-visible spectrophotometer) at $490 \mathrm{~nm}$. Readings were tabulated and calibration curve was prepared.

(2) Sample Preparation and Analysis of Total Sugar Content in Fruit Juice Samples. About $10 \mathrm{~mL}$ of fruit nectar samples was centrifuged at $4000 \mathrm{rpm}$ for 20 minutes. Then $10^{4}$ times dilute solution was prepared. Total sugar content was measured according to the above procedure.

2.5. Comparative Study of Lane Eynon Titration Method and Spectrophotometric Method. Total sugar content of fruit nectar samples was obtained using both methods: Lane and Eynon titration method and spectrophotometric method. Results were statistically analyzed using SAS 9.0 software at $\alpha=0.05$. Study design was Randomized Complete Block Design (RCBD).

\subsection{Determination of Synthetic Colorants in Fruit Nectars}

2.6.1. Sample Preparation and Colorant Extraction from Fruit Juices. Fruit juice sample was centrifuged at $2500 \mathrm{rpm}$ for 15 minutes. Then $5 \mathrm{~mL}$ from upper layer was acidified using $10 \mathrm{~mL}$ of $2 \mathrm{M}$ glacial acetic acid [15]. Then the sheep-wool was put into the conical flask and placed in water bath at $100^{\circ} \mathrm{C}$ for $1 \mathrm{hr}$ and 30 minutes. Then the sheep-wool was taken out and washed under running tap water for 10 minutes. $2 \mathrm{M}$ ammonia solution was added to color containing sheep-wool and placed in water bath. When all colors were released from sheep-wool to ammonia solution, the sheep-wool was removed. Temperature of the water bath was maintained at $100^{\circ} \mathrm{C}$ to evaporate all ammonia from mixture. Then, the concentrated color was used for further analysis.

2.6.2. Identification of the Extracted Colorants of Fruit Juice Samples. The extracted colorants were identified using thin-layer chromatography (TLC) method. n-Butanol: water : glacial acetic acid $(20: 12: 10 \mathrm{v} / \mathrm{v})$ system was used. Standard colors and extracted colors were dissolved in $60 \%$ ethanol. Samples were spotted with standards (Carmosine (E122), Ponceau 4R (E124), Allura Red (E129), Sunset Yellow FCF (E110), Tartrazine (E102), and some nonpermitted colors in food industry as Fast Red E, Ponceau S, Sudan, and Metanil Yellow) in the same TLC plate [16]. Retention factor $\left(R_{\mathrm{f}}\right)$ values of each sample were calculated and compared with color standards. $R_{\mathrm{f}}$ values were calculated for all standards and samples.

\section{Results and Discussion}

In this research, the consumer survey was conducted to collect the information on consumer preference of the fruit juice products, awareness level, and opinion about the color cording system for sugar introduced by the food regulatory authority in Sri Lanka. It was conducted throughout the country to elicit information on consumption pattern of 
three beverages (fruit nectars, carbonated beverages, and cordial) among Sri Lankans. Based on the survey, fruit juice brands and product types were selected for further analysis.

Similar approaches were done in the following researches. Rambukwella et al. [1] carried out a research using a pretested questionnaire to determine the consumer preference of Fruit Drinks and Carbonated Drinks in Sri Lankan Beverage Market. In this research, random samples of 600 respondents were taken from Gampaha, Anuradhapura, Hambantota, and Kandy municipal council areas. The main objective of the study was to identify the existing consumer preference of carbonated drinks and fruit drinks in the Sri Lankan beverage market. The other objectives were to find out the factors that influence the consumption of carbonated drinks and fruit drinks and to study the consumer preference for the various existing brands of fruit drinks [1].

3.1. Sociodemographic Characteristics of the Respondents. A total of 220 responded to this questionnaire survey. Based on the sociodemographic characteristics of the responders, $51 \%$ of target population were male and $49 \%$ were female. The majority of the respondents $(74 \%)$ are $15-25$ years of age. The lowest, $6 \%$, reported from above is 50 years of age category. $12 \%$ and $8 \%$ were recorded from 36 to 50 years and 26-35 years, respectively. But, for some of the questions, only a part of 220 responded.

3.2. The Consumption Pattern of Beverages. According to Figure 1, the majority of respondents consumed fruit nectar (47\%) and 17\% preferred fruit cordial. Second highest (36\%) preference was carbonated beverages.

According to the findings of Rambukwella et al. [1], more than $75 \%$ of the consumers in all four districts where they conducted the study preferred fruit drinks over carbonated drinks because they considered them healthy. However, consumers used the majority of the carbonated drinks because they are having a good taste, higher availability (everywhere), convenience, and relatively less cost than fruit drinks [1].

According to our research finding, fruit nectar consumption is considerably higher than carbonated beverages and fruit cordial among Sri Lankans. Unit price of these products is also lower than cordial and can purchase smallsized (buddy) packs. Consumer can purchase these products from supermarkets, grocery shops, and small retailer shops located all over the country at any time. It can be concluded that consumers prefer more to purchase fruit nectars to quench their thirst and it has a higher contribution to the Recommended Dietary Allowance (RDA) of sugar. An important finding that emerged out of the survey was that $47 \%$ of the respondents approve the popularizing of the fruit drink industry in Sri Lanka instead of carbonated drinks.

Figure 2 shows the frequency consumption of beverages. 214 persons out of the 220 study population responded frequency of fruit nectar consumption and 212 persons

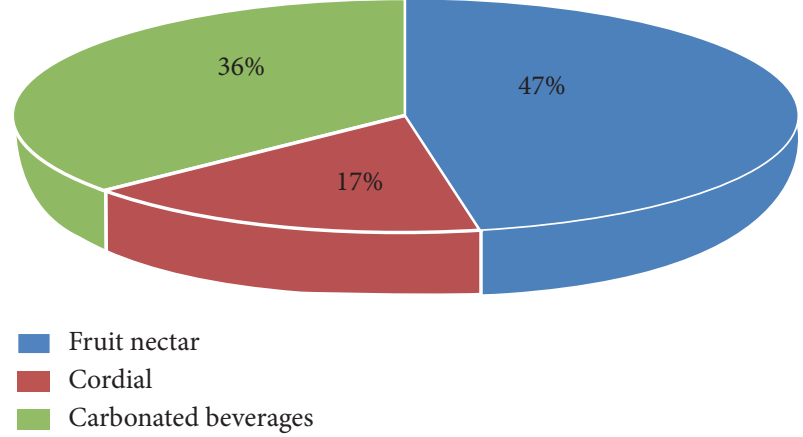

Figure 1: Consumer preference of beverages.

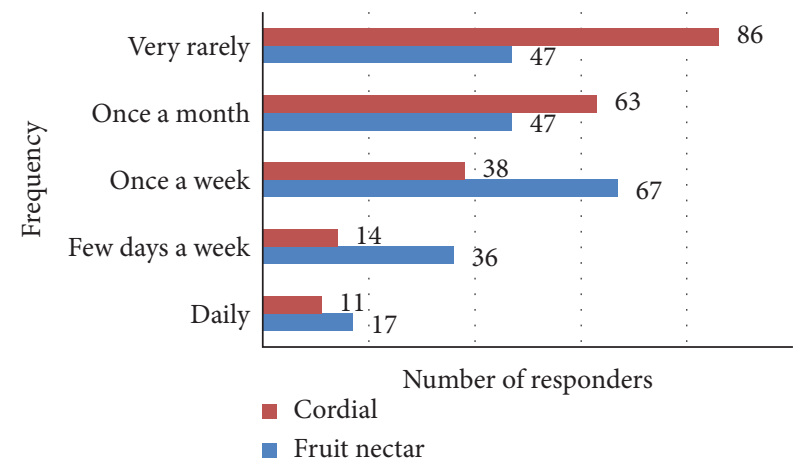

Figure 2: Frequency of consumption of beverages.

out of the 220 study population responded frequency of cordial consumption in this consumer survey. According to Figure 2, most of the people (67) consumed fruit nectars once a week and cordial was consumed very rarely (86). This consumption pattern explains the popularity of fruit nectar among the respondents than cordial.

According to Figure 3, the majority of the respondents (population size $=220$ ) preferred brand 2 while $2^{\text {nd }}$ and $3^{\text {rd }}$ preferences were given to brands 1 and 3 , respectively. Some of the respondents preferred more than one brand. Therefore, they selected more than one brand at once in the consumer survey. Most popular 4 brands of fruit nectars were purchased and named using a number system for laboratory analysis to maintain the confidentiality.

Consumer preference on product type is given in Figure 4 . The majority of the respondents (107) out of total respondents (220) preferred mixed fruit nectar (48.63\%). $2^{\text {nd }}, 3^{\text {rd }}$, and $4^{\text {th }}$ preferences were recorded for mango (82), orange (67), and wood apple (52) fruit nectars, respectively. Only 5 respondents preferred Nelli (Phyllanthus emblica) fruit nectar. Most of the respondents selected more than one product type as their preference.

According to Figure 5, ninety percentage $(90 \%)$ of the respondents usually consumed one bottle $(200 \mathrm{~mL})$ at a time. But $2 \%$ of the target population were taking more than 4 bottles at once.

3.3. Consumer Awareness on Sugar RDA. Sugar sweetened beverages, such as fruit nectars and undiluted/diluted form 


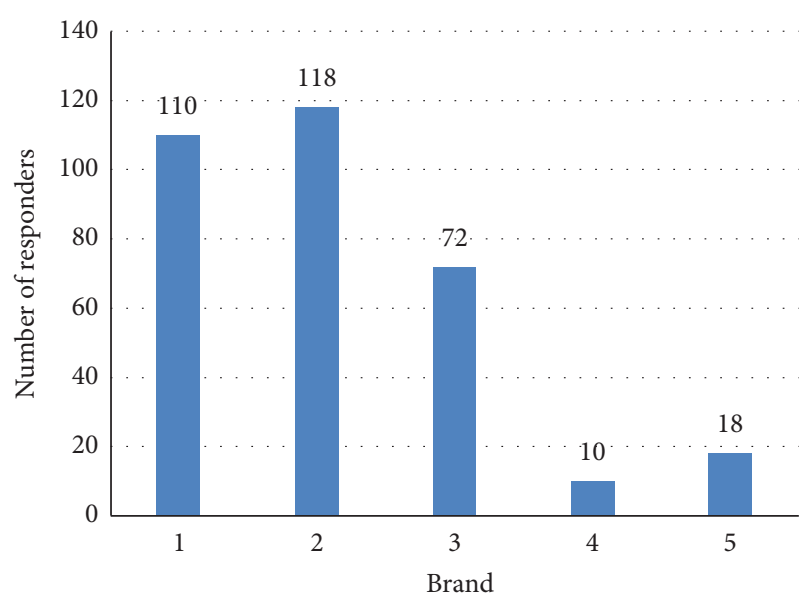

FIGURE 3: Distribution of preference for the popular brands.

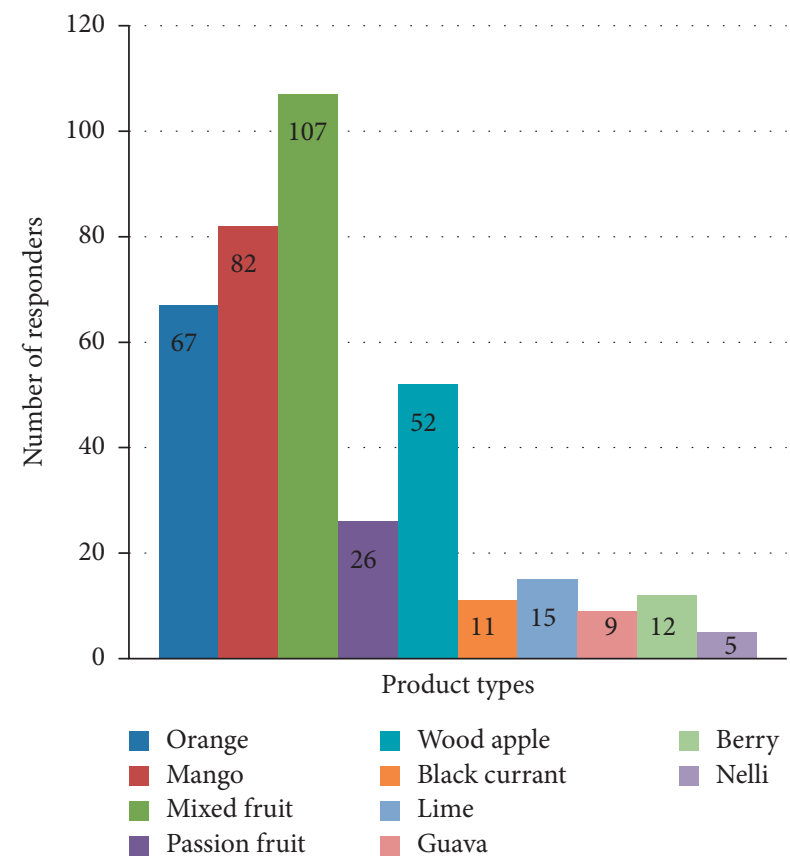

FIGURE 4: Distribution of preference of fruit nectars.

of squashes, contain higher level of added sugar. Therefore, they have lesser health benefit. Normally, in this type of fruit juices a single cup contains the recommended daily amount of sugar for adults and exceeds the recommendations for children [2]. Researchers have found that there is a relationship between higher intake of sugar and increased risk of obesity, cardiovascular disease including dyslipidemia, elevated blood pressure as well as diabetes, non-alcoholic fatty liver disease, cognitive decline, and cancer [17]. Therefore, this research finds that consumer awareness is very important to minimize health risk of the people.

Figure 6 shows the consumer awareness on Recommended Dietary Allowance (RDA). 103 persons out of the 220 target population answered this question during the consumer survey. From that $49 \%$ of the target population were aware about RDA of sugar and $51 \%$ of the population did not have an idea about it. From that 48 stated RDA of

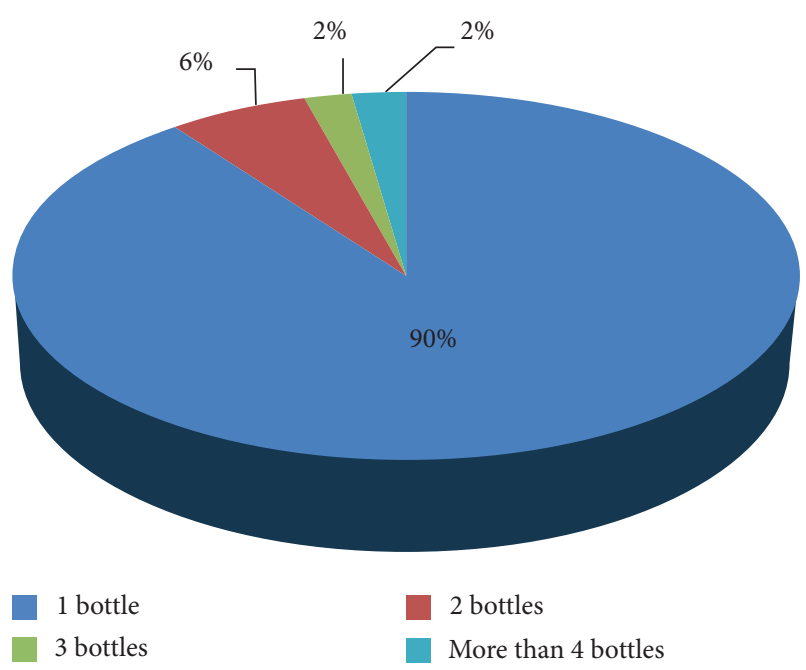

FIGURE 5: Number of bottles consumed per one purchase (fruit nectar).

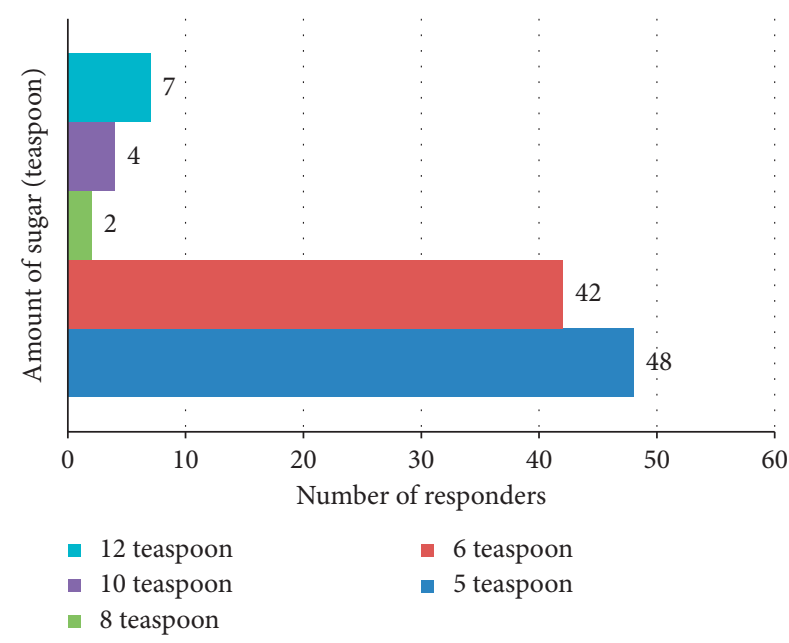

FIgURE 6: Consumer awareness on RDA value of sugar $(12 \mathrm{tsp}=50.00 \mathrm{~g}, 10 \mathrm{tsp}=41.67 \mathrm{~g}, 8 \mathrm{tsp}=33.33 \mathrm{~g}, 6 \mathrm{tsp}=25.00 \mathrm{~g}$, $5 \mathrm{tsp}=20.83 \mathrm{~g}$ ).

sugar was 5 teaspoon per day, and 42 stated it was 6 teaspoon per day. According to the WHO recommendation, RDA value of sugar is 6 teaspoons $(25.0 \mathrm{~g})$ [4]. The American Heart Association (AHA) recommends limiting amount of added sugar; according to this recommendation daily intake sugar for a male is $37.5 \mathrm{~g}$ (9 tea spoons) and for a female, $25.0 \mathrm{~g}$ (6 tea spoons) [18].

Results of the factor considering at purchasing by consumers were taste $(37 \%)$, price $(28 \%)$, brand $(28 \%)$, and the nutritional value (7\%), respectively, in target population (220 respondents).

According to the findings of other studies, more than $80 \%$ of the consumers are not concerned about the ingredients of the beverages but $92 \%$ of the respondents are concerned only about the date of expiry. According to the statistical relationship, taste was the only factor that was considered by the sample respondents when they consumed both fruit and carbonated drinks. Furthermore, it was 
mentioned that appearance and package were not considered much by the target population. Fruit drink market in Sri Lanka is dominated by well-reputed brands of local fruit drinks, followed by well-reputed brands of imported fruit drinks. Hence, small-scale fruit drinks limited only to particular localities cannot compete in this context. However, there is a demand for that variety from rural areas of all four districts [1].

Therefore, the results revealed that the main decisive factor is the taste while the least influencing factor was the nutritional value of the product. Brand name and price of the product equally affected the choice of a beverage.

Some of the local population (51\%) are unaware of RDA value of sugar added sugar consumption and relationship with the risk of chronic disease. Therefore, less attention is given to the nutritional value of the products during purchasing of beverages. This could be the major reason for receding that over $40 \%$ of the Sri Lankan population were prediabetic or diabetic. The alarming situation is that more than $13 \%$ of children, some below 12 years, are being diagnosed as prediabetic [5]. Therefore, awareness on safe sugar level and available sugar content of beverages and other foods is of utmost importance for the people in Sri Lanka.

3.4. Awareness on Traffic Light Color Coding System. The majority of consumers had a good impression about traffic light system (color coding system) used in fruit-based beverages. $68 \%$ of target population of the consumer survey know well this color coding system. According to their comments, it can be identified as very convenient and beneficial method for the consumer. Because it is a visual method, no need to spend time to read labels. Color can be identified easily and gives an idea quickly. This method is beneficial not only to the diabetic patients, but also to all consumers.

3.5. Total Sugar Content in Fruit Nectars. Total sugar content in fruit nectars analyzed by both the Lane and Eynon method and spectrophotometric method is given in Table 1 . According to the Lane and Eynon titration method, maximum level of total sugar level $(18.38 \pm 0.01 \mathrm{~g} / 100 \mathrm{~mL})$ was observed in wood apple nectar in brand No. " 1 ," whereas the minimum total sugar content $(9.17 \pm 0.01 \mathrm{~g} / 100 \mathrm{~mL})$ was identified in mixed fruit nectar in brand No. "2". According to the spectrophotometric method, maximum level of total sugar level $(18.31 \pm 0.02 \mathrm{~g} / 100 \mathrm{~mL})$ was observed in wood apple nectar in brand No. "1," whereas the minimum total sugar content $(9.23 \pm 0.12 \mathrm{~g} / 100 \mathrm{~mL})$ was identified in mixed fruit nectar in brand No. "2."

3.6. Sucrose Content in Fruit Nectars. According to Table 2, maximum sucrose content was observed in mango nectar in the brand No. "1" and the value was $10.57 \mathrm{~g} / 100 \mathrm{~mL}$ sucrose out of $17.39 \mathrm{~g} / 100 \mathrm{~mL}$ total sugar. The lowest sucrose content was observed in wood apple nectar in the brand No. " 3 " and the value was $1.16 \mathrm{~g} / 100 \mathrm{~mL}$ sucrose out of $13.79 \mathrm{~g} / 100 \mathrm{~mL}$ total sugar.

\subsection{Comparative Study of Lane and Eynon Titration Method and Spectrophotometric Method}

3.7.1. Statistical Analysis. Total sugar contents of both test methods were compared using SAS 9.0 (Randomized Complete Block Design, RCBD). There is no significant difference between the two testing methods $(P>0.05)$.

\subsubsection{Comparison of Two Laboratory Tested Methods.} The phenol-sulfuric acid method (spectrophotometric method) is a simple and rapid colorimetric method to determine total carbohydrates in a sample. The method detects virtually all classes of carbohydrates, including monosaccharides, disaccharides, oligosaccharides, and polysaccharides. Under the high temperature and extremely low $\mathrm{pH}$, carbohydrates are hydrolyzed and produce furan derivative compounds, which react with phenolic compounds and produce colored substances. These colored substances absorb light in the visible range, with a maximum absorbance at a wavelength of $490 \mathrm{~nm}$ because phenol forms colored complexes and these complexes absorb light in the visible range, with a maximum absorbance at a wavelength of $490 \mathrm{~nm}[19]$.

Similar approaches were used in other studies. According to the research on Spectrophotometry as a Tool for Dosage Sugars in Nectar of Crops Pollinated by Honeybees, this method is used to identify the simple or complex sugars and their derivatives, including methyl esters with free reducing groups or potentially free, when treated with phenol and concentrated sulfuric acid resulting yellow-orange color. This reaction is sensible and the color is stable [20].

When comparing titration method, sample preparation procedure of spectrophotometric method is somewhat complicated. In this method sample should be prepared very purely; if particles are present, it can affect the absorbance value. Sensitivity of this method ranges from 10 to $100 \mu \mathrm{g} / \mathrm{mL}$ of total sugar [19]. So, samples were diluted $10^{4}$ times for taking absorbance value from this range. Results obtained from the spectrophotometer and calculation procedure of spectrophotometric method are comparatively convenient than titration method.

Although it is easier to use than many of the available methods, the phenol-sulfuric acid method has a few serious drawbacks. The coloring agent used in this method, phenol, causes multiple health hazards. Phenol and its vapors are corrosive to skin, eye, and respiratory system. Repeated and prolonged contact with skin can cause dermatitis or secondand third-degree burns. Similarly, prolonged or repeated inhalation of phenol vapors causes lung edema. Long-term exposure to phenol also has serious impacts on the central nervous system (it is a strong neurotoxin), kidneys, and liver. Result of the standard phenol-sulfuric acid method is presented in terms of glucose-equivalent concentrations. This representation may have potential limitations when dealing with complex carbohydrates that are not simple polymers of glucose. Chemical reactivity of carbohydrates with the derivatization reagent (sulfuric-acid) greatly depends on 
TABLE 1: Total sugar content in fruit nectars (Lane and Eynon titration method and spectrophotometric method).

\begin{tabular}{|c|c|c|c|}
\hline \multirow{2}{*}{ Brand } & \multirow{2}{*}{ Product } & \multicolumn{2}{|c|}{ Total sugar content $(\mathrm{g} / 100 \mathrm{~mL})$} \\
\hline & & Lane and Eynon titration method & Spectrophotometric method \\
\hline \multirow{4}{*}{1} & Mango & $17.39 \pm 0.01$ & $17.8 \pm 0.21$ \\
\hline & Mixed fruit & $9.68 \pm 0.03$ & $10.52 \pm 0.65$ \\
\hline & Wood apple & $18.38 \pm 0.01$ & $18.31 \pm 0.02$ \\
\hline & Orange & $11.82 \pm 0.12$ & $11.72 \pm 0.01$ \\
\hline \multirow{3}{*}{2} & Mango & $9.68 \pm 0.05$ & $9.32 \pm 0.05$ \\
\hline & Mixed fruit & $9.17 \pm 0.01$ & $9.23 \pm 0.12$ \\
\hline & Wood apple & $12.25 \pm 0.01$ & $12.72 \pm 0.37$ \\
\hline \multirow{4}{*}{3} & Mango & $12.24 \pm 0.01$ & $12.04 \pm 0.01$ \\
\hline & Mixed fruit & $11.69 \pm 0.03$ & $11.79 \pm 0.01$ \\
\hline & Wood apple & $13.79 \pm 0.01$ & $13.26 \pm 0.33$ \\
\hline & Orange & $13.62 \pm 0.24$ & $13.03 \pm 0.04$ \\
\hline \multirow{3}{*}{4} & Mango & $12.08 \pm 0.12$ & $12.08 \pm 0.04$ \\
\hline & Mixed fruit & $11.57 \pm 0.10$ & $12.2 \pm 0.68$ \\
\hline & Orange & $11.82 \pm 0.11$ & $11.87 \pm 0.16$ \\
\hline
\end{tabular}

TABLE 2: Sucrose content in fruit nectars (Lane and Eynon titration method).

\begin{tabular}{lccc}
\hline Brand & Product & Sucrose $(\% \mathrm{w} / \mathrm{w})$ & Sucrose content $(\mathrm{g} / 100 \mathrm{~mL})$ \\
\hline \multirow{4}{*}{1} & Mango & 10.27 & 10.57 \\
& Mixed fruit & 5.42 & 5.58 \\
& Wood apple & 7.54 & 7.77 \\
\hline & Orange & 6.34 & 6.57 \\
\hline & Mango & 5.86 & 5.94 \\
& Mixed fruit & 5.40 & 5.47 \\
& Wood apple & 6.77 & 6.74 \\
\hline & Mango & 5.78 & 5.97 \\
& Mixed fruit & 6.30 & 6.45 \\
& Wood apple & 1.13 & 1.16 \\
& Orange & 1.36 & 1.38 \\
& Mango & 5.05 & 5.15 \\
& Mixed fruit & 7.88 & 8.07 \\
\end{tabular}

whether the carbohydrates are neutral or anionic. As a result, the molar absorption coefficients can vary greatly depending on the charge of the carbohydrates analyzed [21].

Both methods are unable to distinguish different types of reducing sugars present in fruit nectar samples. Concentration of nonreducing sugars cannot be determined by using spectrophotometric method. So, when comparing both methods, the titration method provides more details than the spectrophotometric method. For the titration method, glassware is only needed, which is generally available in laboratories and there is no need for specific equipment. When considering colorimetric method, UV-Visible spectrophotometer is required for the analysis. This type of equipment is expensive. So, titration method can be identified as a cost-effective method.

According to Figure 7, total sugar content obtained from Lane and Eynon titration method was higher than label values in most of the products other than mixed fruit and mango nectar in brand No. " 2 " and mixed fruit nectar in brand No. "1." The highest deviation from label value to titration value was observed in mango nectar in brand No.
"1." Second and third highest deviations were observed in orange nectar in brand No. "3" and wood apple nectar in brand No. "1," respectively.

When considering spectrophotometric method (colorimetric method), most of the products other than mixed fruit and mango nectar in brand No. " 2 " had a high sugar level with compared to the label value. The highest deviation from label value to spectrophotometric method value was observed in mango nectar in brand No. "1." Second and third highest deviations were observed in wood apple nectar in brand No. " 1 " and orange nectar in brand No. "3," respectively.

Comparing the analyzed data and the color coding system, introduced by the Extra Ordinary gazette, except mixed fruit in brand-1, mango, and mixed fruit in brand-2, all other types of fruit nectar should have the red color code since these samples contain above $11 \mathrm{~g}$ of sugar per $100 \mathrm{~mL}$ of the sample. However, according to our laboratory analyzed data, orange type in brand-1, wood apple in brand-2, mango, mixed fruit, and orange in brand- 3 and mango, mixed fruit, and orange in brand-4 should have the red 


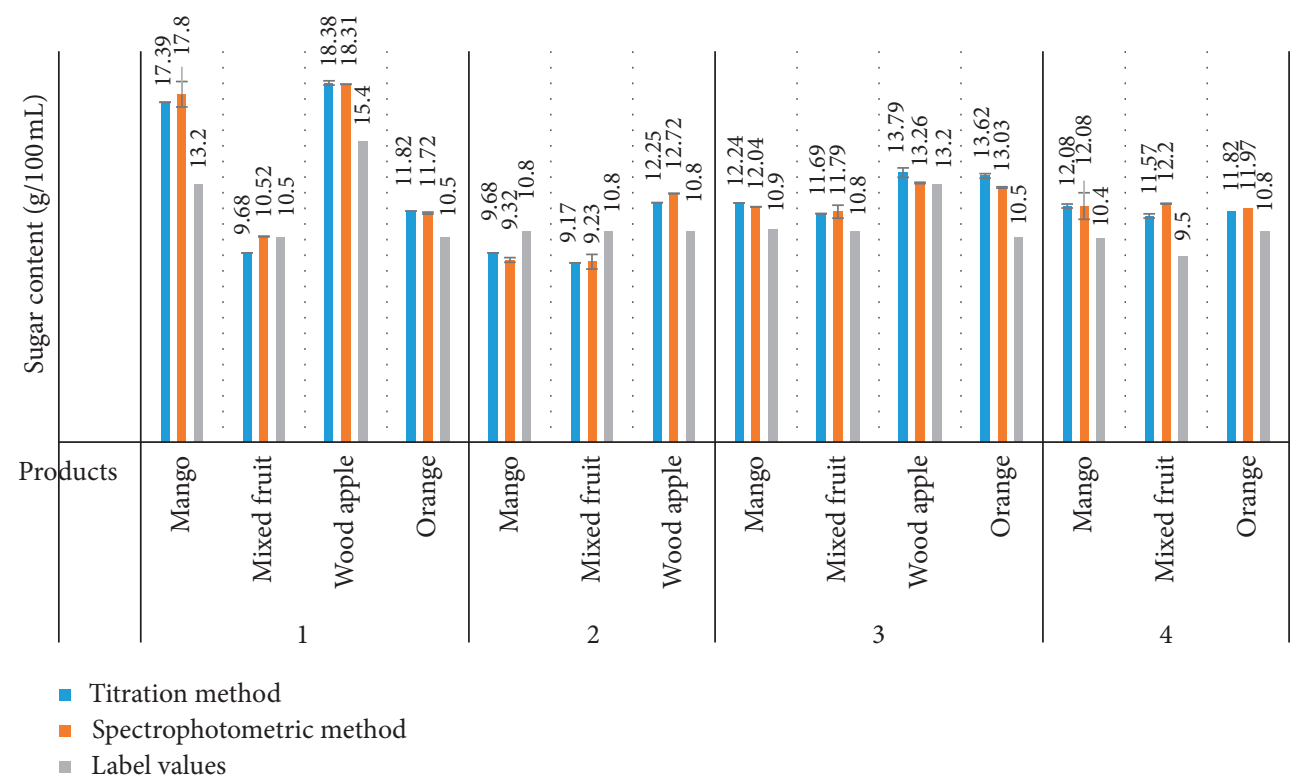

FIGURE 7: Comparison of the titration method values and spectrophotometric method (colorimetric method) values with products label values.

TABLE 3: Behavior of standard synthetic colors on TLC with $n$-butanol : water: glacial acetic acid $(20: 12: 10 \mathrm{v} / \mathrm{v})$ solvent system.

\begin{tabular}{lcc}
\hline Color standard & $R_{\mathrm{f}}$ value & Color of the spot \\
\hline Carmosine & 0.79 & Dark strawberry red \\
Ponceau 4R & 0.47 & Strawberry red \\
Fast red E & 0.67 & Dark red \\
Ponceau S & 0.39 & Strawberry red \\
Allura red & 0.70 & Orange red \\
Sunset yellow & 0.74 & Orange \\
Tartrazine & 0.55 & Lemon yellow \\
Metanil yellow & 0.87 & Yellow \\
Sudan 1 & 0.95 & Light yellow \\
Sudan 2 & 0.94 & Light orange \\
Sudan 3 & 0.93 & Light orange \\
Sudan 4 & 0.93 & Light red \\
\hline
\end{tabular}

color codes although manufactures labeled these all in amber color. Therefore, 8 products $(57.14 \%)$ out of total samples (14 products from 4 brands) violated the Sri Lankan regulations.

This research finding provides an idea that the food manufacturers are not following regulations properly on color code system according to the sugar level, under the Traffic Lights Color Coding system regulation No. 1965/18 (August, 2016), of the Food Act No. 26 of 1980. Product labels are also not accurate. Therefore, consumers are misled by the labels.

3.8. Identification of Synthetic Colorants in Fruit Nectars by Using Thin-Layer Chromatography. Table 3 shows retention factor $\left(R_{\mathrm{f}}\right)$ values of selected permitted and some nonpermitted colorants.

According to our finding, a total of 14 product types were analyzed and the majority of fruit nectar samples (57.14\%) contained natural colorants. Only $42.86 \%$ of total samples contained synthetic colorants. All the synthetic colorants were permitted according to the Food Act No. 26 of 1980, Extra ordinary Gazette No. 1688/28, 2011.

According to the laboratory analyzed data given in Table 4, mango, mixed fruit, and wood apple nectar in brand-2 and mango, mixed fruit, and orange nectar in brand -4 contain sunset yellow. Mango nectar in brand- 2 and mango nectar in brand-4 contain tartrazine. Only mixed fruit nectar in brand-2 contains carmoisine. Allura red, ponceau $4 \mathrm{R}$, and methyl yellow were not found in selected fruit nectar samples.

When comparing the laboratory analyzed data with the label information, synthetic colorants found in all the products from brand- 4 were matching with label information. However, synthetic colorants found in all the products from brand- 2 were not mentioned in label information. Therefore, 3 products $(21.43 \%)$ out of total samples (14 products from 4 brands) violated the Sri Lankan regulations. 
TABLE 4: Different types of synthetic and natural colorants present in fruit nectar samples.

\begin{tabular}{|c|c|c|c|}
\hline Brand & Product & $R_{\mathrm{f}}$ value & Types of colorant present \\
\hline \multirow{4}{*}{1} & Mango & & Natural colorant (carotenes) \\
\hline & Mixed fruit & & Natural colorant (carotenes) \\
\hline & Wood apple & & Natural colorant (caramel and ammonia caramel) \\
\hline & Orange & & Natural colorant (carotenes) \\
\hline \multirow{5}{*}{2} & Mango & $R_{\mathrm{f} 1}=0.74$ & \\
\hline & & $R_{\mathrm{f} 2}=0.55$ & syntmetic cororant (sunsel yenow and tartrazme) \\
\hline & Mixed fruit & $R_{\mathrm{f} 1}=0.74$ & Synthetic colorant (sunset yellow and carmoisine) \\
\hline & & $R_{\mathrm{f} 2}=0.79$ & 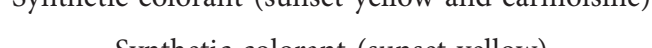 \\
\hline & Wood apple & $R_{\mathrm{f}}=0.74$ & Synthetic colorant (sunset yellow) \\
\hline \multirow{4}{*}{3} & Mango & & Natural colorant (carotenes) \\
\hline & Mixed fruit & & Natural colorant (carotenes) \\
\hline & Wood apple & & Natural colorant (caramel) \\
\hline & Orange & & Natural colorant (carotenes) \\
\hline \multirow{3}{*}{4} & Mango & $\begin{array}{l}R_{\mathrm{f} 1}=0.74 \\
R_{\mathrm{f} 2}=0.55\end{array}$ & Synthetic colorant (sunset yellow and tartrazine) \\
\hline & Mixed fruit & $R_{\mathrm{f}}=0.74$ & Synthetic colorant (sunset yellow) \\
\hline & Orange & $R_{\mathrm{f}}=0.74$ & Synthetic colorant (sunset yellow) \\
\hline
\end{tabular}

\section{Conclusion}

In our study, method comparison in sugar analysis was conducted between the Lane and Eynon titration method and UV-visible spectrophotometric method. There was no significant different between the results of the two analytical methods at $\alpha=0.05$. When considering advantages and disadvantages of methods, the Lane and Eynon titration method is identified as the most recommended method for total sugar analysis in fruit juice samples. The average sugar content in analyzed fruit nectars varies from 18.38 to $9.17 \mathrm{~g} / 100 \mathrm{~mL}$. Hence, normally $100 \mathrm{~mL}$ can be recommended to consume per day with taking other sugar containing food items.

Total sugar level with respect to both analytical methods was higher than the label values indicated in most of the products $(57.14 \%)$ other than mixed fruit and mango nectar in brand No. "2." The majority of food manufacturers (57.14\%) did not adhere to the "Traffic Light" color coding system and labelling regulations correctly. Therefore, this research finding shows the necessity of the constant vigilance to ensure that the food manufactures comply with the regulations of food colors and color coding for sugar level.

Majority of fruit nectar samples contained natural colorants. Some of the samples contained permitted synthetic colorants. $21.43 \%$ samples out of total samples violated the label regulation. This research finding provides useful information for preparation and implementation in national policies and for updating Sri Lankan food act and regulations to improve the quality of foods and drinks in Sri Lanka, thus improving the health condition of the community.

\section{Data Availability}

All the raw data are available with the corresponding author.

\section{Disclosure}

This research was done as a collaborative study between the Department of Nutrition, Medical Research Institute,
Colombo 08, and the Department of Food Science and Technology, Faculty of Agriculture, University of Peradeniya, Sri Lanka. This research did not receive specific funding but was performed as part of the employment of the authors.

\section{Conflicts of Interest}

The authors declare that there are no conflicts of interest.

\section{Acknowledgments}

The authors express their gratitude to the Director and staff members of the Department of Nutrition, Medical Research Institute, Colombo 08, for providing comfortable working environment to conduct this research. Furthermore, the authors are grateful to the staff members of Department of Food Science and Technology, Faculty of Agriculture, University of Peradeniya, for the great support given throughout this research.

\section{References}

[1] R. Rambukwella, D. Priyadarshana, and R. Vidanapathirana, "Comparative study on consumer preference of fruit drinks and carbonated drinks in Sri Lankan beverage market research," 2015, http://www.harti.gov.lk/images/download/ reasearch_report/new1/177.pdf.

[2] R. G. Duffett, "Consumption patterns and demographic factors influence on fruit juice classifications, health benefits and sugar content perceptions in two Municipal Districts in Cape Town, Western Cape, South Africa," South African Journal of Clinical Nutrition, vol. 31, no. 1, pp. 20-28.

[3] The Food (Colour Coding for Sugar Levels) Regulations, The Gazette of the Democratic Socialist Republic of Sri Lanka Extraordinary, No. 1965/18, Sri Lanka, 2016.

[4] WHO Calls on Countries to Reduce Sugars Intake among Adults and Children, 2018, https://www.who.int/mediacentre/ news/releases/2015/sugar-guideline/en/. 
[5] Labels that Hide the Poison, 2018, http://srilankabrief.org/ 2016/08/suger-in-food-drinks-labels-that-hide-the-poison/.

[6] A. Mortensen, "Carotenoids and other pigments as natural colorants," Pure and Applied Chemistry, vol. 78, no. 8, pp. 1477-1491, 2006.

[7] L. Koutsogeorgopoulou, C. Maravelias, G. Methenitou, and A. Koutselinis, Immunological Aspects of the Common Food Colorants, Amaranth and Tartrazine, Department of Forensic Medicine And Toxicology, School of Medicine, Athens, Greece, 1998.

[8] T. M. Fonovich, "Sudan dyes: are they dangerous for human health?," Drug and Chemical Toxicology, vol. 36, no. 3, pp. 343-352, 2013.

[9] B. A. Geoffrey and M. B. Felix, "Canthaxanthin and the eye: a critical ocular toxicologic assessment," Journal of Toxicology: Cutaneous and Ocular Toxicology, vol. 10, no. 1-2, pp. 115155, 1991.

[10] D. M. Hinton, "US FDA “redbook II" immunotoxicity testing guidelines and research in immunotoxicity evaluations of food chemicals and new food proteins," Toxicologic Pathology, vol. 28 , no. 3, pp. 467-478, 2000.

[11] J. C. Larsen, "Erythrosine toxicological evaluation of certain food additives and contaminants," in Proceedings of the 37th meeting of JECFA-WHO. Food Additives Series, vol. 28, pp. 171-180, Geneva, Switzerland, 1991.

[12] The Food (Colouring Substances) Regulations, The Gazette of the Democratic Socialist Republic of Sri Lanka Extra Ordinary No. 1688/28, 2011.

[13] Survey to Consumer Demand for Fruit Nectar and Cordial, https://docs.google.com/forms/d/e/1FAIpQLSe2nPtrxp fLSdK75XxgxccOAFh6P7Xo9tpRvs92U5mOccGcsg/viewform.

[14] "Method 923.09: invert sugar in sugars and syrups, laneeynon general volumetric method, final action," in Official Methods of Analysis of Association of Official Analytical Chemists, Better World Books, Reno, NV, USA, 15th edition, 1990.

[15] F. Farzianpour, J. G. Khaniki, M. Younesian, B. B. Ghahferkhi, M. Sadeghi, and S. Hosseini, "Evaluation of food color consumption and determining color type by thin layer chromatography," American Journal of Applied Sciences, vol. 10, no. 2, pp. 172-178, 2013.

[16] G. K. M. Rajapaksha, M. A. J. Wansapala, and A. B. G. Silva, "Determination of synthetic colors in selected food and beverages available in Colombo district, Sri Lanka," International Journal of Science and Research, vol. 6, pp. 801-808, 2015.

[17] J. Rippe and T. Angelopoulos, "Relationship between added sugars consumption and chronic disease risk factors: current understanding," Nutrients, vol. 8, no. 11, p. 697, 2016.

[18] Daily Intake of Sugar-How Much Sugar Should You Eat Per Day?, 2018, https://www.healthline.com/nutrition/how-muchsugar-per-day.

[19] M. DuBois, K. A. Gilles, J. K. Hamilton, P. A. Rebers, and F. Smith, "Colorimetric method for determination of sugars and related substances," Analytical Chemistry, vol. 28, no. 3, pp. 350-356, 1956.

[20] V. A. D. Toledo, M. C. Ruvolo-Takasusuki, A. Oliveira, E. Chambó, and S. Mara Sanches Lopes, Spectrophotometry as a Tool for Dosage Sugars in Nectar of Crops Pollinated by Honeybees, In Tech, Philadelphia, PA, USA, 2012.

[21] M. Mecozzi, "Estimation of total carbohydrate amount in environmental samples by the phenol-sulphuric acid method assisted by multivariate calibration," Chemometrics and Intelligent Laboratory Systems, vol. 79, no. 1-2, pp. 84-90, 2005. 